\title{
Negative regulatory effect of an oligodendrocytic bHLH factor OLIG2 on the astrocytic differentiation pathway
}

\author{
S Fukuda ${ }^{1,2,4}$, T Kondo $^{\star 1,4,5}$, H Takebayashi $^{3}$ and T Taga ${ }^{\star, 1,2}$ \\ ${ }^{1}$ Department of Cell Fate Modulation, Institute of Molecular Embryology and \\ Genetics, Kumamoto University, Japan \\ 2 The 21st Century COE Program 'Cell Fate Regulation Research and \\ Education Unit', Kumamoto University, Kumamoto 860-0811, Japan \\ ${ }^{3}$ Division of Molecular Neurobiology, National Institute for Physiological \\ Sciences, 38 Nishigonaka, Myodaiij, Okazaki 444-8585, Japan \\ 4 These two authors contributed equally to this work. \\ ${ }^{5}$ Current address: Centre for Brain Repair, University of Cambridge, The E.D. \\ Adrian Building, Forvie Site, Robinson Way, Cambridge CB2 2PY, UK. \\ * Corresponding authors: T Taga and T Kondo, Department of Cell Fate \\ Modulation, Institute of Molecular Embryology and Genetics, Kumamoto \\ University, Kumamoto 860-0811, Japan. Tel: + 81-96-373-6610; \\ Fax: + 81-96-373-6614; E-mail: taga@kaiju.medic.kumamoto-u.ac.jp (TT) \\ and tk294@cam.ac.uk (TK)
}

Received 23.6.03; revised 01.9.03; accepted 01.9.03; published online 24.10.03 Edited by $\mathrm{H}$ Ichijo

\begin{abstract}
In the developing vertebrate nervous system, multipotent neural stem cells produce both neurons and glia. OLIG2 is a basic helix-loop-helix transcription factor that plays critical roles in oligodendrocyte and motor neuron development; however, its role in astrocytic development remains elusive. In this study, we analyzed an effect of OLIG2 on cytokineinduced astrocytic differentiation from mouse telencephalic neuroepithelial cells. We show that the presence of OLIG2 protein leads to inhibition of the promoter activation of astrocyte-specific glial fibrillary acidic protein gene. We found that OLIG2 abolishes complex formation between a transcriptional coactivator $\mathrm{p} 300$ and a transcription factor, signal transducer and activator of transcription 3 (STAT3), which is activated by astrocytic differentiation-inducing cytokines, such as leukemia inhibitory factor (LIF). The enforced expression of OLIG2 in neuroepithelial cells inhibits the LIF-induced astrocytic differentiation. We also show that the OLIG2 protein in the nuclei of neural precursor cells disappears in accordance with astrocytic differentiation during culture with LIF. Together, these results reveal a novel molecular function of OLIG2 on the astrocyte development.

Cell Death and Differentiation (2004) 11, 196-202. doi:10.1038/ sj.cdd. 4401332

Published online 24 October 2003
\end{abstract}

Keywords: OLIG2; p300; STAT3; cytokine; neuroepithelial cell; astrocytic differentiation

\footnotetext{
Abbreviations: STAT3, signal transducer and activator of transcription 3; LIF, leukemia inhibitory factor; GFAP, glial fibrillary acidic protein; bHLH, basic helix-loop-helix; GFP, green fluorescent protein; bFGF, basic fibroblast growth factor
}

\section{Introduction}

Mouse fetal telencephalic neuroepithelial cells give rise to neurons, astrocytes and oligodendrocytes, ${ }^{1-3}$ whose differentiation is thought to be regulated by cell-external cues and cell-intrinsic programs. ${ }^{4,5}$ One of such cell-external cues is interleukin-6 (IL-6) family of cytokines that have various biological effects on cell growth, differentiation and survival in multiple cell types. ${ }^{6}$ IL- 6 and members of this family, that is, IL-11, leukemia inhibitory factor (LIF), ciliary neurotrophic factor, oncostatin $M$, cardiotrophin-1 and cardiotrophin-like cytokine, are pleiotropic and display overlapping functions. For example, all of them have a potential to induce astrocytic differentiation from neuroepithelial cells, which involves activation of a downstream transcription factor signal transducer and activator of transcription (STAT3). ${ }^{7-12}$

In the nervous system, bone morphogenetic protein 2 (BMP2), a member of the transforming growth factor- $\beta$ superfamily, is also involved in astrocytic differentiation. ${ }^{8,13}$ BMP2-induced heterodimerization of its receptors activates downstream transcription factors Smad1, -5 and -8 , and these Smad proteins interact with the common Smad, Smad4. Similar to STAT3, activated Smad proteins also translocate to the nucleus and activates specific target genes. ${ }^{14}$

Previously, we have shown that two cytokines, LIF and BMP2, act in synergy on primary fetal neural progenitor cells to induce astrocytes. ${ }^{8}$ These cytokines activate respective downstream transcription factors, STAT3 and Smad1, which are bridged by a transcriptional coactivator p300 in the nucleus. The formation of this ternary complex is prerequisite for the differentiation of glial fibrillary acidic protein (GFAP)positive astrocytes from undifferentiated neuroepithelial cells.

In addition to these transcriptional regulators, various members of the basic helix-loop-helix (bHLH) protein family also play essential roles in the generation of specific subsets of neurons. ${ }^{15-18}$ Two bHLH transcription factors, OLIG1 and OLIG2, were identified as proteins regulating oligodendrocyte development. ${ }^{19-21}$ OLIG2 has also been shown to play an essential role in the generation of motor neurons. ${ }^{21-23}$ Interestingly, in mice deficient for both OLIG1 and OLIG2, astrocytes came out ectopically in the region of ventral spinal cord where OLIG2 is normally expressed. ${ }^{24}$ These results raised a possibility that OLIG2 and/or OLIG1 may have a negative regulatory effect on astrocytic development.

We here show that OLIG2 has inhibitory effects on astrocytic differentiation and that OLIG2 prevents formation of the STAT3-p300 complex that is known to be required for the development of astrocytes. Finding of the antiastrocytic property of oligodendrocytic factor OLIG2 suggests the presence of a negative interaction between astrocytic and oligodendrocytic differentiation pathways. 


\section{Results \\ OLIG2 represses GFAP gene transcription in neuroepithelial cells}

We wanted to know whether an oligodendrocytic transcription factor OLIG2 has a potential to inhibit astrocytic differentiation. We first examined whether OLIG2 could suppress the expression of an astrocyte-specific protein, GFAP. Neuroepithelial cells were transfected with a GFAP promoterluciferase reporter vector together with or without an OLIG2 expression vector, and then stimulated with LIF, which is known to induce astrocytic differentiation. As shown in Figure 1, LIF stimulation induced transcriptional activation of the GFAP promoter. By contrast, forced expression of OLIG2 significantly suppressed this activation. OLIG2 did not exhibit negative effect on a promoter for elongation factor $1 \alpha$ in the internal control plasmid, pEF-Rluc (data not shown). These results suggest that OLIG2 can specifically block the expression of GFAP at the transcriptional level.

\section{OLIG2 binds to p300 and abolishes the complex formation between STAT3 and p300}

Since we have previously shown that LIF stimulation activates STAT3 and allows it to form a complex with p300 in the nucleus, which is essential for GFAP expression, ${ }^{8}$ we hypothesized that OLIG2 could interfere with this association, leading to the inhibition of GFAP expression. We wanted to know whether OLIG2 interferes with physical association between STAT3 and p300. We first investigated whether OLIG2 could interact directly with either STAT3 or p300. As shown in Figure 2a, when epitope-tagged OLIG2 and p300 were coexpressed in COS-7 cells, OLIG2 was coimmunoprecipitated with p300 (Figure 2a). Interaction between OLIG2 and STAT3 was not observed (data not shown). To exclude the possibility that the association between OLIG2 and p300 is a consequence of overexpression, we examined whether this

relative GFAP promoter activation

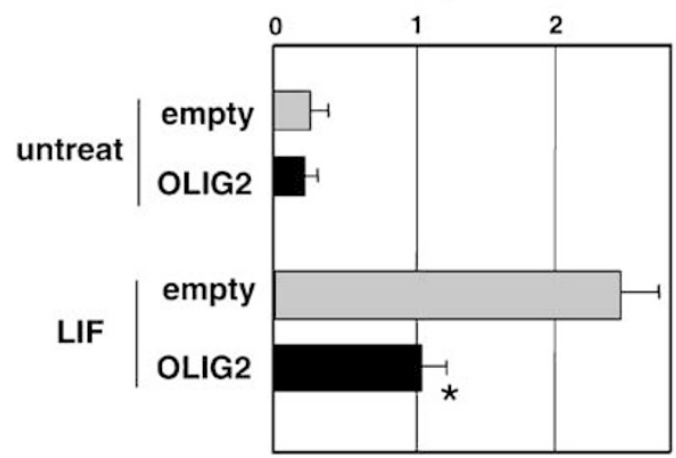

Figure 1 Inhibition of GFAP promoter activation by OLIG2. Neuroepithelia cells were transfected with GF1L-pGL3 and pEF-Rluc, together with pCMV-HA (control vector; gray bars) or HA-OLIG2-pCMV (OLIG2 vector; black bars) as indicated. After $16 \mathrm{~h}$ incubation, cells were either untreated or treated with $80 \mathrm{ng} /$ $\mathrm{ml}$ of LIF for $8 \mathrm{~h}$ and solubilized. The activity of firefly luciferase was normalized with sea pansy luciferase. An asterisk means statistical significance from the control by Student's $t$-test $(P<0.02)$ a
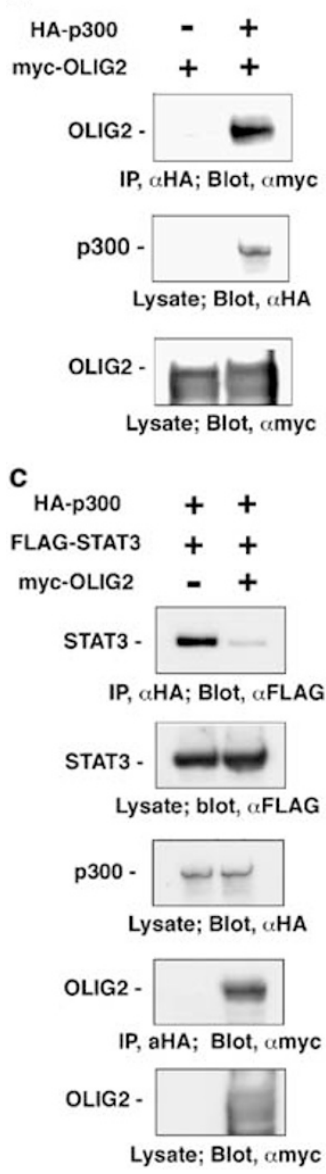

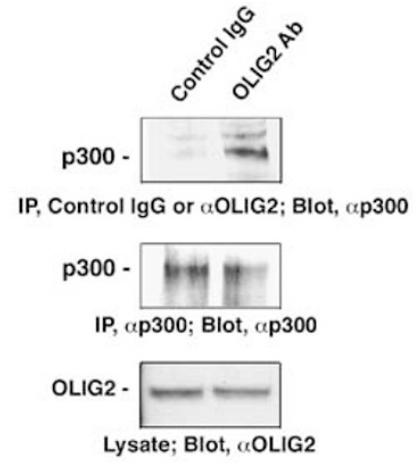

d

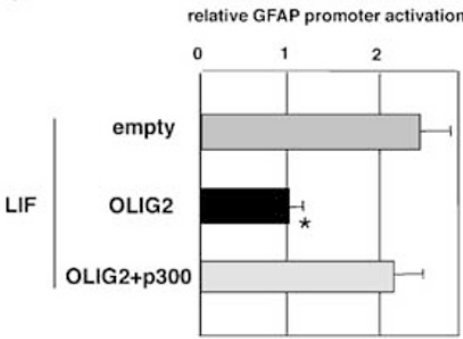

Figure 2 Inhibitory effect of OLIG2 protein on the formation of the STAT3-p300 complex. (a) COS-7 cells were transfected with 6myc-OLIG2-pcDNA3, together with either HA-p300-BOSE or empty vector, as shown on top. After $24 \mathrm{~h}$ incubation, cell lysates were subjected to immunoprecipitation. Immunocomplexes were analyzed by standard SDS-PAGE and Western blotting methods, using antibodies to respective tags. (b) Whole-cell lysates of neuroepithelial cells were subdivided into two tubes and immunoprecipitated with either normal rabbit IgG (control, left lane) or affinity purified anti-OLIG2 antibody (right lane). Immunoprecipitates were analyzed using anti-p300 antibody. (c) COS-7 cells were transfected with HA-p300-BOSE and FLAG-STAT3-BOSE, together with either 6myc-OLIG2-pcDNA3 or empty vector, and analyzed as in (a). (d) Transfection was performed as in Figure 1 except that HA-p300-pCMV $\beta$ was also used (light gray bar). The amount of empty vector included in each transfection was adjusted so that the total amount of the plasmids becomes even. After treatment with LIF for $8 \mathrm{~h}$, luciferase activity was measured

association occurs in the fetal telencephalic neuroepithelial cells. As shown in Figure 2b, endogenous p300 protein was found to associate with endogenous OLIG2 protein in the neuroepithelial cells. These results indicate that OLIG2 binds to 300 in vivo and suggest that OLIG2 may have an inhibitory effect on the STAT3-p300 complex formation. To examine this possibility, p300 and STAT3 were coexpressed either in the absence or presence of OLIG2 in COS-7 cells. In the absence of OLIG2, STAT3 was coimmunoprecipitated with p300 (Figure 2c, left lane). By contrast, in the presence of OLIG2, the interaction between STAT3 and p300 was clearly inhibited (Figure 2c, right lane). These results suggest that OLIG2 can block GFAP gene expression by sequestering p300 from 
STAT3. Thus, we hypothesized that the inhibition of LIF-induced GFAP gene expression by OLIG2 in Figure 1 could be recovered by forced expression of p300. As shown in Figure 2d, the expression of p300 relieved LIFinduced GFAP promoter activation from OLIG2-mediated inhibition.

\section{A region containing the bHLH motif in OLIG2 and both $\mathrm{N}$ - and $\mathrm{C}$-termini of $\mathrm{p300}$ are involved in the physical association between OLIG2 and p300}

To understand the interaction between OLIG2 and p300 in more detail, we mapped protein-protein-interaction domains using deletion constructs. When OLIG2 mutants and a fulllength p300 were coexpressed in COS-7 cells, the middle region of OLIG2 (97-223 amino acids) that contains the bHLH domain was found to bind to p300, whereas either $\mathrm{N}$ - or Cterminal fragments of OLIG2 did not (Figure 3a). This result is reminiscent of previous findings that the bHLH motif in transcription factors, MyoD and BETA2/NeuroD1, interacted with $\mathrm{p} 300 .{ }^{25,26}$ It might thus be possible that a bHLH region in

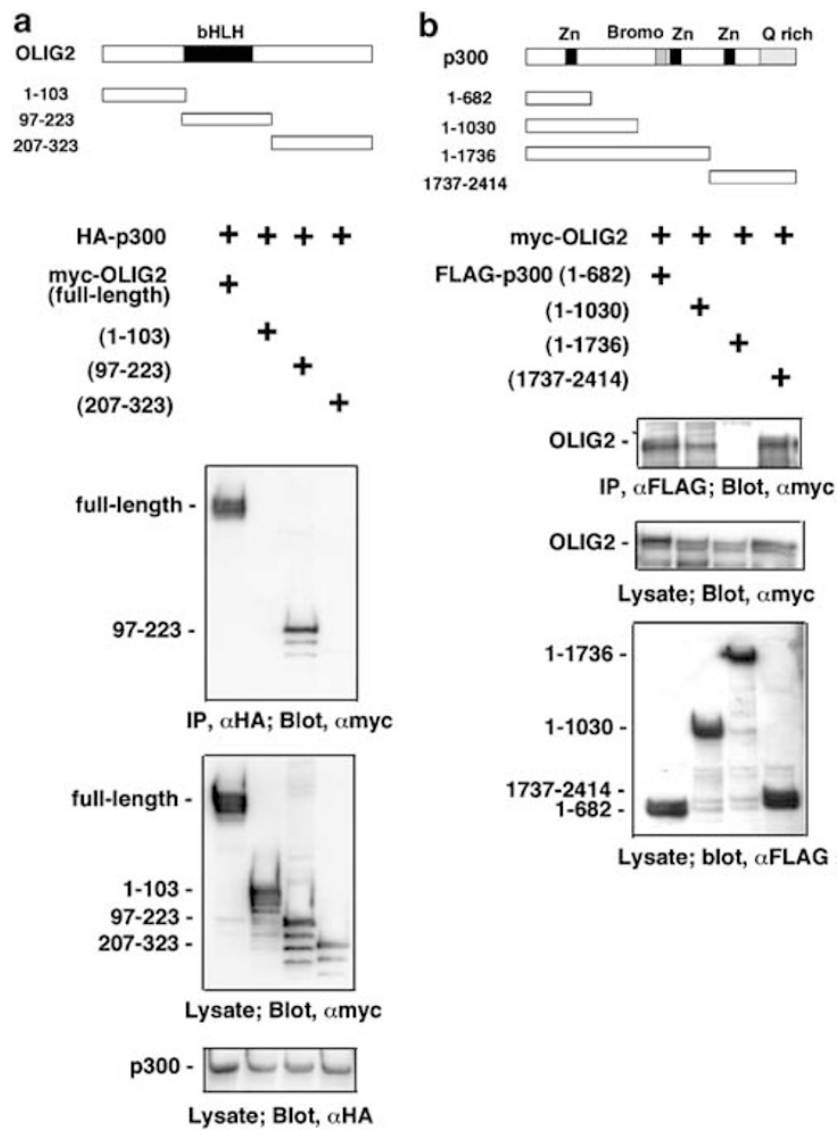

Figure 3 Mapping of the regions responsible for the binding between OLIG2 and $\mathrm{p300}$. (A) Series of plasmids that express myc-tagged OLIG2 deletion mutants and HA-p300-BOSE were introduced into COS-7 cells, and analyzed as in Figure 2. (B) Deletion mutants of FLAG-tagged p300 were tested for its binding activity to myc-tagged full-length OLIG2. Mouse anti-FLAG monoclonal antibody was used for immunoprecipitation. Zn: zinc-finger motif; Bromo: bromodomain; Q-rich: glutamine-rich region some transcription factors may generally interact with p300. In our experimental system, however, forced expression of only the middle region of OLIG2 could not inhibit LIF-induced GFAP promoter activation (data not shown), suggesting that other regions of OLIG2 is also involved in the execution of inhibitory function of OLIG2. We observed extensive protein degradation of the OLIG2 deletion constructs in COS-7 cells, which did not occur in the case of full-length OLIG2 (Figure 3a). The reason how this happened remains unknown. The instability of the OLIG2 fragments might affect the transcriptional repression capability of the OLIG2 deletion mutants.

We then examined which region in the p300 protein interacts with OLIG2. From the experiment with $\mathrm{p} 300$ deletion mutants and full-length OLIG2 expressed in COS-7 cells, we concluded that both $\mathrm{N}$ - and C-termini of p300 had a potential to interact with OLIG2 (Figure $3 b$ ). Similar binding property was observed in the association between STAT3 and p300, in which both $\mathrm{N}$ - and $\mathrm{C}$-termini of p300 interacted with STAT3, although the former showed higher affinity. ${ }^{8}$ It is of interest to note that transcription factors SF-1 and Ets- 1 can also bind to both $\mathrm{N}$ - and C-termini of p300. ${ }^{27,28}$ The fragment of p300 spanning 1-1736 amino-acid residues did not bind to OLIG2 (Figure 3b, lane 3), which implies that the region between residues 1030 and 1736 may inhibit the interaction by masking the $\mathrm{N}$-terminally located OLIG2-interaction domain.

\section{Enforced expression of OLIG2 inhibits astrocytic differentiation}

To examine whether OLIG2 can block astrocytic differentiation, we produced a recombinant OLIG2 retrovirus that encodes both OLIG2 and green fluorescent protein (GFP) genes. Neuroepithelial cells were infected with either a control virus encoding GFP alone or the OLIG2 virus, and then cultured for 4 days with or without LIF. Coexpression of HAtagged OLIG2 and GFP within a single OLIG2 virus-infected neuroepithelial cell by internal ribosome entry site (IRES) was confirmed by immunocytochemistry using anti-HA monoclonal antibody and anti-GFP polyclonal antibody (data not shown). In the absence of LIF, cells infected with either the control or the OLIG2 viruses were GFAP negative (data not shown). In the presence of LIF, $32 \pm 6 \%$ of the control virusinfected GFP-expressing cells were positive for GFAP (Figure 4 , left panel). By contrast, only $22 \pm 4 \%$ of the OLIG2 virus-infected GFP-expressing cells were GFAP positive (Figure 4, right panel). The difference between these two percentages was statistically significant by the Student's $t$-test $(P<0.02)$. The result suggests that OLIG2 can inhibit astrocytic differentiation. In the presence of LIF, the proportion of microtubule-associated protein 2 (MAP2)-positive cells in the OLIG2 virus-infected cells $(8 \pm 2 \%)$ was not significantly different from that in the control virus-infected cells $(7 \pm 3 \%)$. Under the same culture condition, platelet-derived growth factor receptor $\alpha$ chain (PDGF receptor $\alpha$ chain)-positive cells were not emerged from neuroepithelial cells infected with either the control virus or the OLIG2 virus (data not shown). 

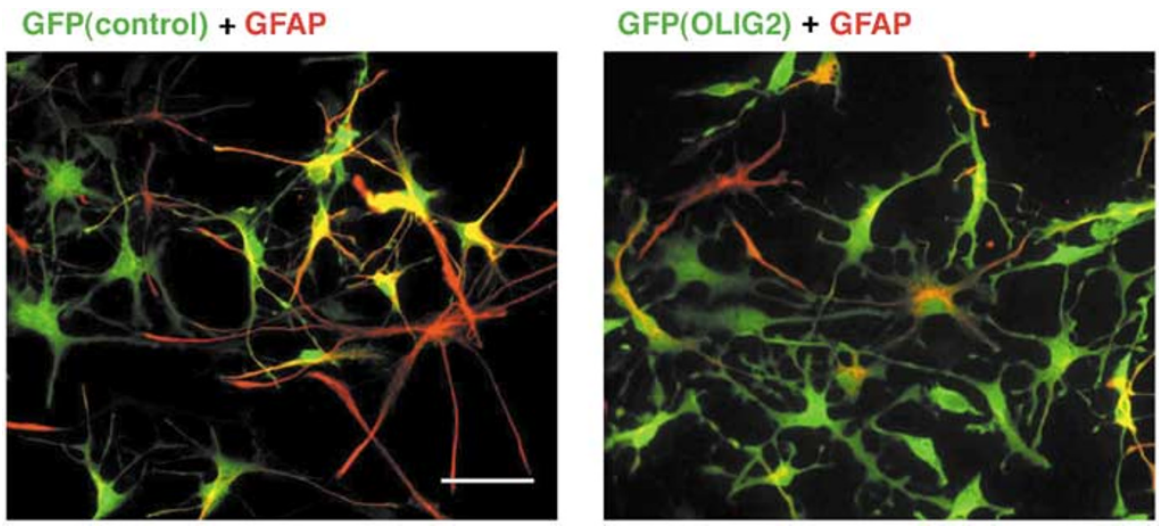

Figure 4 Effect of forced expression of OLIG2 on astrocytic differentiation. Neuroepithelial cells were infected with a recombinant retrovirus that encodes both OLIG2 and GFP (right panel). A retrovirus that encodes GFP alone was used as a control (left panel). At $24 \mathrm{~h}$ after infection, the medium was replaced with the fresh medium containing $80 \mathrm{ng} / \mathrm{ml}$ of LIF. After 4 days, cells were fixed and stained with anti-GFP polyclonal antibody (green) and anti-GFAP monoclonal antibody (red). Scale bar, $30 \mu \mathrm{m}$

\section{Endogenous OLIG2 protein disappears from the nucleus in differentiated astrocytes}

Now, it is consistent that a single multipotent neural stem cell can generate neurospheres in the presence of basic fibroblast growth factor (bFGF) and differentiate into oligodendrocytes and astrocytes, as well as neurons. ${ }^{29}$ The results described above raise a question whether expression levels of OLIG2 are downregulated during astrocytic differentiation. To answer this question, we examined the expression of OLIG2 in neuroepithelial cells by immunocytochemistry. Neuroepithelial cells which had been cultured for 4 days were replated on chamber slides and stained with anti-OLIG2 antibody. ${ }^{21}$ As shown in Figure $5 a$ and $b$, we found that nuclei of $91 \pm 2 \%$ the cell population were positive for OLIG2 on the following day of replating on chamber slides just prior to LIF stimulation (day 0 , Figure $5 a$ and $b$ ). To examine the relationship between OLIG2 and GFAP expression, these cells were cultured with or without LIF, and stained with anti-OLIG2 and anti-GFAP antibodies. When the cells were treated with LIF for 4 days, $30 \pm 6 \%$ of cells were positive for the expression of GFAP, and these cells showed negligible OLIG2 immunoreactivity in their nuclei, in marked contrast to GFAP-negative cells (Figure 5c and d). In cells cultured without LIF for 4 days, GFAP-positive cells were barely detectable, and such cells, if any, expressed OLIG2 in their nuclei, whose level was comparable to that of GFAP definitely negative cells (Figure $5 \mathrm{e}$ and $\mathrm{f}$ ). These results indicated that downregulation of OLIG2 expression is presumably necessary for neuroepithelial cells to undergo astrocytic differentiation in culture.

\section{Discussion}

A large number of transcription factors have been found to regulate neural cell fates, either positively or negatively, and some of them can induce differentiation of one cell lineage while inhibiting another. We and others have shown that a reciprocal mechanism operates between neurogenesis and astrocytogenesis: BMP2 induces the development of astrocytes, and at the same time suppresses neurogenesis by inhibiting neurogenic bHLH transcription factors via the expression of Id1, Id 3 and Hes-5 proteins. ${ }^{30}$ By contrast, a bHLH transcription factor neurogenin (Ngn1) promotes neurogenesis, and at the same time inhibits astrocytogenesis by inhibiting STAT3 ${ }^{31}$ Here, we have shown a novel negative interaction between astrocytic and oligodendrocytic differentiation signals: OLIG2, which was originally thought to be involved in the development of motor neurons and oligodendrocytes, also blocks astrocytogenesis by inhibiting complex formation between p300 and STAT3. This finding of the dual function of OLIG2 in gliogenesis is consistent with the observation that astrocytes are ectopically generated instead of oligodendrocytes in the spinal cord of OLIG1/2 double knockout mice. ${ }^{24}$ Incompleteness in the inhibition of astrocytic differentiation by OLIG2 overexpression may partly be because OLIG2 requires a cooperative factor (or factors) for full inhibitory activity, which might include OLIG1. Recently, another astrocytic differentiation cytokine, BMP4, was shown to inhibit the expression of OLIG2 ${ }^{32}$ This finding also supports the idea of the presence of mutual negative interactions between astrocytic and oligodendrocytic differentiation pathways. As we showed previously, ${ }^{8}$ a BMP2 downstream transcription factor Smad1 forms a complex with STAT3 and p300. No physical interaction between Smad1 and OLIG2 was observed (data not shown), but the effects of OLIG2 on the Smad1-p300 complex formation remains to be elucidated.

The present results showing the interaction of OLIG2 with a transcriptional coactivator $\mathrm{p} 300$ raise a possibility that OLIG2 may function as a transcriptional activator in neural cell development. However, this seems unlikely since a previous report showed that the bHLH domain of OLIG2 fused to the VP16 transactivation domain significantly inhibited motor neuron development. ${ }^{23}$ We suppose that OLIG2 would have two distinct aspects as a transcriptional repressor, that is, transcriptional suppression by recruiting an unidentified corepressor protein complex and by sequestering coactivator p300.

In vivo, OLIG2 is expressed in the restricted bilateral ventral regions of the neural tube from which motor neurons and oligodendrocytes are generated. It should be noted, however, that once the neuroepithelium is triturated and cultured on a dish, almost all neuroepithelial cells express OLIG2 (Figure 5a 


\section{OLIG2 + GFAP}
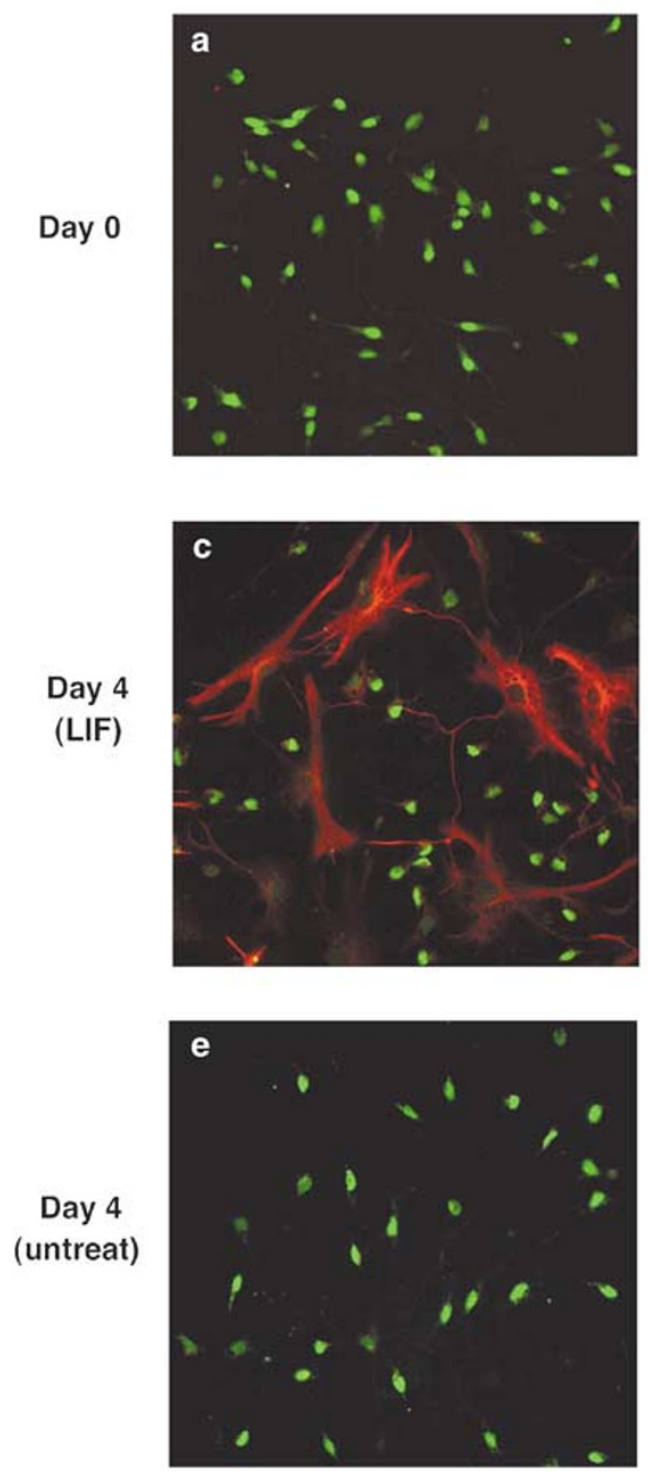

Hoechst + GFAP
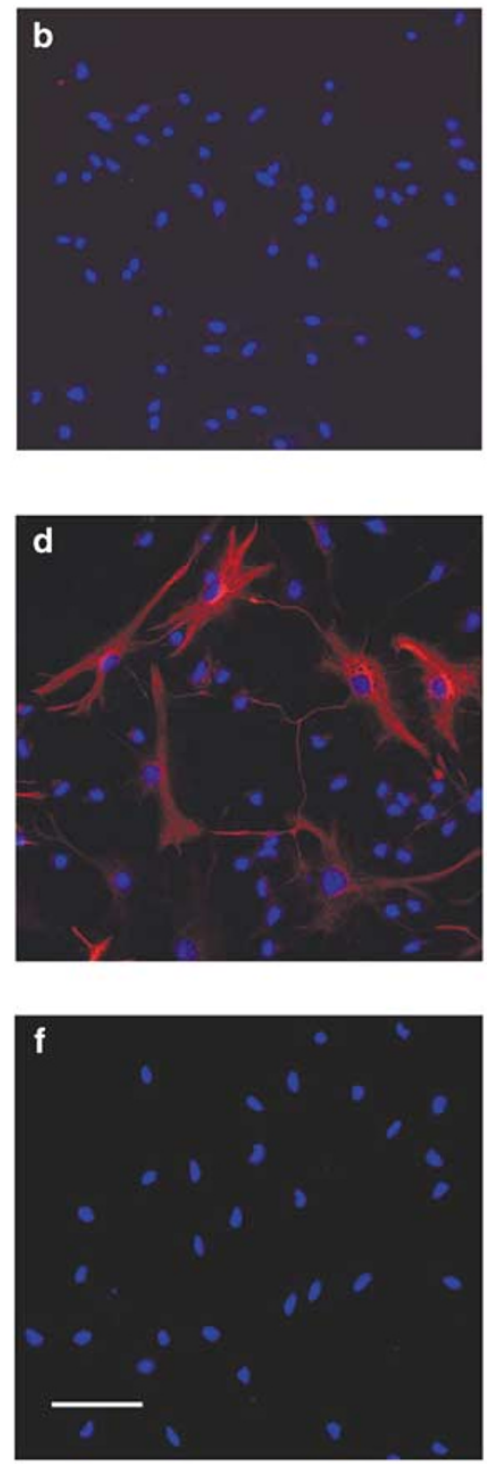

Figure 5 Disappearance of OLIG2 protein from the nucleus in differentiated astrocytes. Neuroepithelial cells were plated on precoated 8-well chamber slides. On the following day, the medium was replaced (day 0 ) and cultured for further 4 days in the presence (c, d) or absence (e, f) of $80 \mathrm{ng} / \mathrm{ml}$ LIF. (a, c, e) Cells were fixed as indicated, and double labeled with anti-OLIG2 polyclonal antibody (green) and anti-GFAP monoclonal antibody (red). (b, d, f) Hoechst nuclear staining views (blue) of the same fields in $(\mathbf{a}, \mathbf{c}, \mathbf{e})$, respectively, were merged with the images of the GFAP staining. Scale bar, $30 \mathrm{~m}$

and $\mathrm{b}$ ), indicating that they might acquire a potential to differentiate into oligodendrocytes in vitro. Dissociation process or monolayer culture with bFGF may affect some regulatory elements that control OLIG2 expression. When the neuroepithelial cells were cultured in the absence of bFGF with $0.1 \%$ fetal calf serum (FCS) for 7 days, $48 \pm 3,20 \pm 4$, $9 \pm 2 \%$ of the cells were immunolabeled for MAP2, GFAP and galactocerebroside, respectively (data not shown). This fact indicates that OLIG2 expression is not sufficient to initiate and progress the oligodendrocytic differentiation pathway in cultured neuroepithelial cells and that some other factors, such as Nkx2.2, should cooperate as reported previously. ${ }^{33}$ Further investigations that identify upstream regulatory elements/factors or binding proteins of OLIG2 would provide valuable clues for fully understanding the role of OLIG2. In addition, these studies would also be of much importance in elucidating the transcription factor network that determines the fate of cells in the developing central nervous system.

\section{Materials and Methods}

\section{Cell culture}

Time-pregnant ICR mice were treated according to the guidelines of Kumamoto University Center for Animal Resources and Development. Neuroepithelial cells were isolated from telencephalons of E14.5 mice and expanded for 4 days in N2-supplemented DMEM/F-12 containing $10 \mathrm{ng} / \mathrm{ml}$ basic fibroblast growth factor (bFGF) as described previously. ${ }^{8}$ Cells were 
then detached and used for further experiments. Mouse LIF (ESGRO, Invitrogen) was used for the induction of astrocytes at a concentration of $80 \mathrm{ng} / \mathrm{ml}$. COS-7 cells were maintained in DMEM supplemented with $10 \%$ FCS. A retrovirus packaging cell line, Plat-E, was maintained in DMEM supplemented with $10 \%$ FCS, $10 \mu \mathrm{g} / \mathrm{ml}$ of blasticidin and $1 \mu \mathrm{g} / \mathrm{ml}$ of puromycin. $^{34}$

\section{Plasmid constructions}

CDNA for mouse OLIG2 was amplified by reverse transcription and polymerase chain reaction (RT-PCR) using Superscript First Strand Synthesis System for RT-PCR (Invitrogen) and Pfu Turbo DNA polymerase (STRATAGENE). Total RNA was isolated by ISOGEN (Nippongene) from mouse neuroepithelial cells and used as a template in RT-PCR. The obtained cDNA was inserted into pcDNA3 (Invitrogen) and pCMV-HA (CLONTECH), resulting in 6myc-OLIG2-pcDNA3 and HAOLIG2-pCMV, respectively. OLIG2 deletion mutants (N-terminus, 1-103 residues; middle region, 97-223 residues; C-terminus, 207-323 residues) were made by a standard subcloning procedure. Firefly luciferase reporter plasmid driven by the $2.5 \mathrm{~kb}$ GFAP gene promoter (GF1L-pGL3), sea pansy luciferase plasmid (pEF-Rluc), expression plasmids of STAT3 (FLAG-STAT3-BOSE), full-length p300 (HA-p300-pCMV $\beta$ and HA-p300BOSE) and a series of FLAG-tagged p300 deletion mutant plasmids were described previously. ${ }^{8}$

\section{Reporter assay}

Neuroepithelial cells, which had been cultured for 4 days with bFGF, were replated on 24-well plates the day before the transfection. Cells were transfected with the reporter plasmid, GF1L-pGL3, and internal control plasmid, pEF-Rluc, together with the expression plasmids as described in figure legends using TransIT-LT1 (Mirus). On the following day, LIF was added to the medium at a concentration of $80 \mathrm{ng} / \mathrm{ml}$, and cells were incubated for $8 \mathrm{~h}$. Luciferase activity was measured using Pikkagene Dual Luciferase Assay System (Tokyo Ink Inc.).

\section{Immunoprecipitation}

COS-7 cells were plated on $60 \mathrm{~mm}$ dishes. On the following day, cells were transfected with the expression plasmids using TransIT-LT1. After $24 \mathrm{~h}$ incubation, cells were solubilized in NP40 lysis buffer $(10 \mathrm{mM}$ Tris at $\mathrm{pH}$ 7.5, $150 \mathrm{mM} \mathrm{NaCl}, 0.5 \%$ NP40, $5 \mathrm{mM}$ EDTA, $5 \mathrm{mg} / \mathrm{ml}$ aprotinine and $3 \mathrm{mM}$ pAPMSF) and cell lysates were cleared by centrifugation. The lysates were subjected to immunoprecipitation with rabbit anti-HA antibody (Santa Cruz, Y-11). Immunocomplexes were analyzed by standard SDS-PAGE and Western blotting methods using anti-HA antibody, mouse anti-FLAG antibody (Sigma, M2) and mouse anti-myc antibody (UPSTATE, 9E10). To detect the interaction between endogenous OLIG2 and p300 proteins, neuroepithelial cells expanded for 4 days were plated on $60 \mathrm{~mm}$ dishes and cultured for further 2 days in the presence of bFGF. Whole-cell lysates were prepared in NP40 lysis buffer using closed-type sonicator (COSMO $\mathrm{BIO}$ ) and immunoprecipitated with either normal rabbit IgG (Santa Cruz, sc-2027) or affinity purified anti-OLIG2 antibody. ${ }^{21}$ Immunoprecipitates were analyzed using anti-p300 antibody (Santa Cruz, C-20).

\section{Immunocytochemistry}

Neuroepithelial cells replated on 8-well chamber slides were fixed in 4\% paraformaldehyde and preincubated in blocking solution (PBS containing $10 \%$ FCS and $0.1 \%$ Triton X-100). Then cells were double stained with mouse anti-GFAP antibody (Sigma, G3893) and rabbit anti-GFP antibody (MBL, 598) diluted in the blocking solution. Anti-MAP2 monoclonal antibody (Simga, M4403) and anti-PDGF receptor $\alpha$ chain (BD Pharmingen, 558774) were also used. Nuclei were stained using Hoechst 33258 (nacalai tesque).

\section{Retroviral production}

For the recombinant retrovirus construction, CDNA for OLIG2 was inserted into pMY-IRES-GFP ${ }^{34}$ and the plasmid was introduced into Plat-E using TransIT-293 (Mirus). After $48 \mathrm{~h}$ incubation, culture medium containing produced recombinant retroviruses was centrifuged, and the collected viruses were resuspended in N2-supplemented DMEM/F-12 containing $10 \mathrm{ng} / \mathrm{ml} \mathrm{bFGF}$.

\section{Acknowledgements}

We thank Dr. K Ikenaka for helpful discussion and Dr. M Raff for critical reading of the manuscript. We also thank Dr. T Kitamura for Plat-E cells, and members of our laboratory for valuable discussions and technical advice. We are very grateful to Ms. Y Noguchi for her secretarial assistance, and also thank Ms. K Kaneko and Ms. Y Saiki for technical help. This work was supported in part by grant-in-aid for 21 st Century COE Research from Ministry of Education, Science and Culture 'Cell Fate Regulation Research and Education Unit'; Scientific Research (B); Specially Promoted Research from the Ministry of Education, Culture, Science, Sports and Technology; Nakajima Foundation; Uehara Memorial Foundation; Japan Brain Foundation; Higo Foundation; Human Frontier Science Program; and the Virtual Research Institute of Aging of Nippon Boehringer Ingelheim.

\section{References}

1. Anderson DJ (2001) Stem cells and pattern formation in the nervous system: the possible versus the actual. Neuron 30: 19-35

2. Gage FH (2000) Mammalian neural stem cells. Science 287: 1433-1438

3. McKay R (1997) Stem cells in the central nervous system. Science 276: 66-71

4. Edlund T and Jessell TM (1999) Progression from extrinsic to intrinsic signaling in cell fate specification: a view from the nervous system. Cell 96: 211-224

5. Nakashima $\mathrm{K}$ and Taga $\mathrm{T}$ (2002) Mechanisms underlying cytokine-mediated cell-fate regulation in the nervous system. Mol. Neurobiol. 25: 233-244

6. Taga T and Kishimoto T (1997) Gp130 and the interleukin-6 family of cytokines. Annu. Rev. Immunol. 15: 797-819

7. Bonni A, Sun $Y$, Nadal-Vicens M, Bhatt A, Frank DA, Rozovsky I, Stahl N, Yancopoulos GD and Greenberg ME (1997) Regulation of gliogenesis in the central nervous system by the JAK-STAT signaling pathway. Science 278: 477-483

8. Nakashima K, Yanagisawa M, Arakawa H, Kimura N, Hisatsune T, Kawabata M, Miyazono K and Taga T (1999) Synergistic signaling in fetal brain by STAT3Smad1 complex bridged by $\mathrm{p} 300$. Science 284: 479-482

9. Yanagisawa M, Nakashima K and Taga T (1999) STAT3-mediated astrocyte differentiation from mouse fetal neuroepithelial cells by mouse oncostatin M. Neurosci. Lett. 269: 169-172

10. Yanagisawa M, Nakashima K, Arakawa H, Ikenaka K, Yoshida K, Kishimoto T, Hisatsune T and Taga T (2000) Astrocyte differentiation of fetal neuroepithelial cells by interleukin-11 via activation of a common cytokine signal transducer, gp130, and a transcription factor, STAT3. J. Neurochem. 74: 1498-1504

11. Ochiai W, Yanagisawa M, Takizawa T, Nakashima $K$ and Taga $T$ (2001) Astrocyte differentiation of fetal neuroepithelial cells involving cardiotrophin-1induced activation of STAT3. Cytokine 14: 264-271 
12. Uemura A, Takizawa T, Ochiai $W$, Yanagisawa $M$, Nakashima $K$ and Taga $T$ (2002) Cardiotrophin-like cytokine induces astrocyte differentiation of fetal neuroepithelial cells via activation of STAT3. Cytokine 18: 1-7

13. Gross RE, Mehler MF, Mabie PC, Zang Z, Santschi L and Kessler JA (1996) Bone morphogenetic proteins promote astroglial lineage commitment by mammalian subventricular zone progenitor cells. Neuron 17: 595-606

14. Massague J and Chen YG (2000) Controlling TGF-beta signaling. Genes Dev. 14: $627-644$

15. Guillemot F, Lo LC, Johnson JE, Auerbach A, Anderson DJ and Joyner AL (1993) Mammalian achaete-scute homolog 1 is required for the early development of olfactory and autonomic neurons. Cell 75: 463-476

16. Ben-Arie N, Bellen HJ, Armstrong DL, McCall AE, Gordadze PR, Guo Q, Matzuk MM and Zoghbi HY (1997) Math1 is essential for genesis of cerebellar granule neurons. Nature 390: 169-172

17. Ma Q, Chen Z, del Barco Barrantes I, de la Pompa JL and Anderson DJ (1998) Neurogenin1 is essential for the determination of neuronal precursors for proximal cranial sensory ganglia. Neuron 20: 469-482

18. Fode C, Gradwohl G, Morin X, Dierich A, LeMeur M, Goridis C and Guillemot $F$ (1998) The bHLH protein NEUROGENIN 2 is a determination factor for epibranchial placode-derived sensory neurons. Neuron 20: 483-494

19. Lu QR, Yuk D, Alberta JA, Zhu Z, Pawlitzky I, Chan J, McMahon AP, Stiles CD and Rowitch DH (2000) Sonic hedgehog-regulated oligodendrocyte lineage genes encoding bHLH proteins in the mammalian central nervous system. Neuron 25: 317-329

20. Zhou Q, Wang S and Anderson DJ (2000) Identification of a novel family of oligodendrocyte lineage-specific basic helix-loop-helix transcription factors. Neuron 25: 331-343

21. Takebayashi H, Yoshida S, Sugimori M, Kosako H, Kominami R, Nakafuku M and Nabeshima $Y$ (2000) Dynamic expression of basic helix-loop-helix Olig family members: implication of Olig2 in neuron and oligodendrocyte differentiation and identification of a new member, Olig3. Mech. Dev. 99: 143-148

22. Mizuguchi R, Sugimori M, Takebayashi $H$, Kosako $H$, Nagao M, Yoshida S, Nabeshima Y, Shimamura K and Nakafuku M (2001) Combinatorial roles of
Olig2 and Neurogenin2 in the coordinated induction of pan-neuronal and subtype-specific properties of motoneurons. Neuron 31: 757-771

23. Novitch BG, Chen Al and Jessell TM (2001) Coordinate regulation of motor neuron subtype identity and pan-neuronal properties by the bHLH repressor Olig2. Neuron 31: 773-789

24. Zhou $Q$ and Anderson DJ (2002) The bHLH transcription factors OLIG2 and OLIG1 couple neuronal and glial subtype specification. Cell 109: 61-73

25. Eckner R, Yao TP, Oldread E and Livingston DM (1996) Interaction and functional collaboration of p300/CBP and bHLH proteins in muscle and B-cell differentiation. Genes Dev. 10: 2478-2490

26. Mutoh H, Naya FJ, Tsai MJ and Leiter AB (1998) The basic helix-loop-helix protein BETA2 interacts with $\mathrm{p} 300$ to coordinate differentiation of secretinexpressing enteroendocrine cells. Genes Dev. 12: 820-830

27. Monte D, DeWitte F and Hum DW (1998) Regulation of the human P450scc gene by steroidogenic factor 1 is mediated by CBP/p300. J. Biol. Chem. 273: 4585-4591

28. Yang C, Shapiro LH, Rivera M, Kumar A and Brindle PK (1998) A role for CREB binding protein and p300 transcriptional coactivators in Ets-1 transactivation functions. Mol. Cell. Biol. 18: 2218-2229

29. Temple S (2001) The development of neural stem cells. Nature 414: 112-117

30. Nakashima K, Takizawa T, Ochiai W, Yanagisawa M, Hisatsune T, Nakafuku M, Miyazono K, Kishimoto T, Kageyama R and Taga T (2001) BMP2-mediated alteration in the developmental pathway of fetal mouse brain cells from neurogenesis to astrocytogenesis. Proc. Natl. Acad. Sci. USA 98: 5868-5873

31. Sun $Y$, Nadal-Vicens M, Misono S, Lin MZ, Zubiaga A, Hua X, Fan G and Greenberg ME (2001) Neurogenin promotes neurogenesis and inhibits glial differentiation by independent mechanisms. Cell 104: 365-376

32. Mekki-Dauriac S, Agius E, Kan P and Cochard P (2002) Bone morphogenetic proteins negatively control oligodendrocyte precursor specification in the chick spinal cord. Development 129: 5117-5130

33. Zhou Q, Choi G and Anderson DJ (2001) The bHLH transcription factor Olig2 promotes oligodendrocyte differentiation in collaboration with Nkx2.2. Neuron 31: 791-807

34. Morita S, Kojima T and Kitamura T (2000) Plat-E: an efficient and stable system for transient packaging of retroviruses. Gene Therapy 7: 1063-1066 